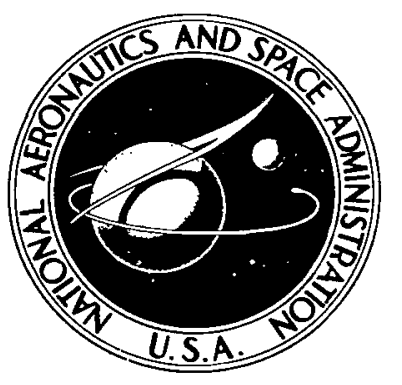

NASA IN D-6214

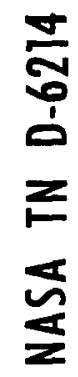

LOAN COPY: REETUI㟒

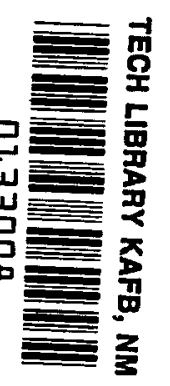

\title{
INVESTIGATION OF INTERMITTENT
}

ENHANCEMENT OF ION EMISSION

FROM A TUNGSTEN SURFACE USING

THE FIELD-ION MICROSCOPE

by Victor G. Weizer

Lewis Research Center

Cleveland, Obio 44135

NATIONAL AERONAUTICS AND SPACE ADMINISTRATION - WASHINGTON, D. C. MARCH 1971 
( $\cdots-1-$

1. Report No.

NASA TN D-6214

4. Title and subtitle INVESTIGATION OF INTERMITTENT ENHANCEMENT OF ION EMISSION FROM A TUNGSTEN SURFACE USING THE FIELD-ION MICROSCOPE

7. Author(s)

Victor G. Weizer

9. Performing Organization Name and Address

Lewis Research Center

National Aeronautics and Space Administration

Cleveland, Ohio 44135

12. Sponsoring Agency Name and Address

National Aeronautics and Space Administration

Washington, D.C. 20546

15. Supplementary Notes
0133008

3. neutpleills varaivy iru.

5. Report Date March 1971

6. Performing Organization Code

8. Performing Organization Report No. E-5511

10. Work Unit No. 129-03

11. Contract or Grant No.

13. Type of Report and Period Covered Technical Note

14. Sponsoring Agency Code

\section{Abstract}

An intermittent enhancement of ion emission or blinking effect is described which is observed when a few parts-per-million neon are added to the imaging gas in a helium-tungsten field-ion microscope. Measurement of the variation of the blinking rate of a single emitting tungsten atom with applied voltage, tip temperature, and gas pressure suggest that (1) adsorption of neon on the apex of a protruding tungsten substrate atom is responsible for the blinking, and (2) the rate of blinking is proportional to $1-\mathrm{P}_{\mathrm{I}}^{++}$, where $\mathrm{P}_{\mathrm{I}}^{++}$is the probability of double ionization as a neon atom passes through the ionization zone above a protruding tungsten emitter.

17. Key Words (Suggested by Author(s)) Field-ion microscopy Adsorption
19. Security Classif. (of this report)

Unclassified
18. Distribution Statement

Unclassified - unlimited

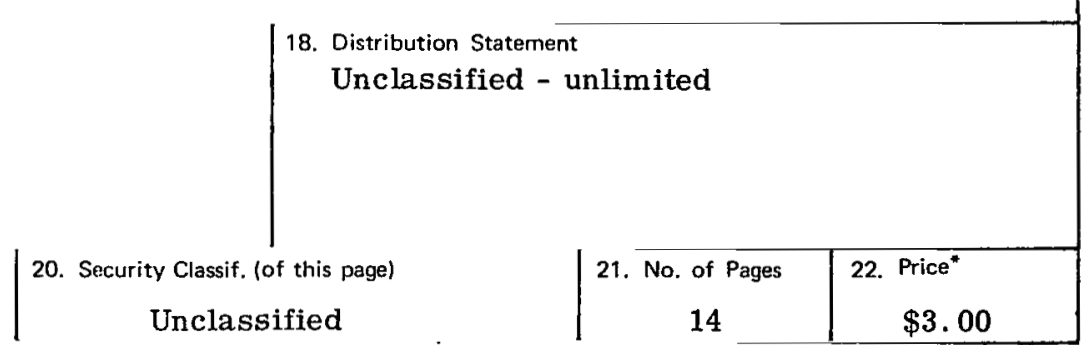

Unclassified
14

$\$ 3.00$

"For sale by the National Technical Information Service, Springfield, Virginia 22151 


\title{
INVESTIGATION OF INTERMITTENT ENHANCEMENT OF ION EMISSION
}

\author{
FROM A TUNGSTEN SURFACE USING THE FIELD-ION MICROSCOPE \\ by Victor G. Weizer
}

Lewis Research Center

\section{SUMMARY}

An intermittent enhancement of ion emission or blinking effect is described which is observed when a few parts-per-million neon are added to the imaging gas in a heliumtungsten field-ion microscope. Measurement of the variation of the blinking rate of a single emitting tungsten atom with applied voltage, tip temperature, and gas pressure suggest that (1) adsorption of neon on the apex of a protruding tungsten substrate atom is responsible for the blinking; and (2) the rate of blinking is proportional to $1-\mathrm{P}_{\mathrm{I}}^{++}$ where $\mathrm{P}_{\mathrm{I}}^{++}$is the probability of double ionization as a neon atom passes through the ionization zone above a protruding tungsten emitter.

\section{INTRODUCTION}

When the tungsten field-ion microscope is operated with helium imaging gas containing a few parts-per-million neon, a random blinking of the normally emitting atoms and their near neighbors can be observed. The voltage range for viewing this effect lies between about 80 and 90 percent of the best image voltage (BIV) at a tip temperature of $5 \mathrm{~K}$.

As the voltage is lowered below about 90 percent BIV, some of the atoms in close proximity to the normally emitting atoms, but which are dark at BIV, begin to emit for short periods of time, after which they return to their original nonemitting state. At a slightly lower voltage, the normally emitting atoms also begin to blink, increasing their brightness momentarily and then returning to normal brightness levels. The total effect on the screen is a random and irregular scintillation.

The purpose of this report is to describe the results of measurements of the characteristic quantities involved in this blinking effect (i.e., the blinking rate, the voltage limits for visibility, etc.), and their variation with temperature, field, and gas pres- 
sure. A theoretical model relating the blinking effect with the probability of neon atom penetration of the ionization zone is proposed which is in agreement with these experimental data.

\section{EXPERIMENTAL RESULTS}

The microscope used in these investigations is essentially the same as described previously (ref. 1). The main feature of the apparatus is that it permits the tip temperature to be varied from $5 \mathrm{~K}$ to room temperature while the imaging gas temperature remains constant. The following modifications, however, were made prior to the start of this work: (1) the original liquid nitrogen cooled screen was exchanged for a room temperature screen to permit direct access to it; and (2) the sample holder was modified as shown in figure 1. Here the tip wire is welded to a nickel-cored copper sleeve that is thermally shrunk-fit on the end of a single crystal quartz rod, the other end of which is attached to the heat exchanger as shown. The $3 / 16$-inch- $(0.476-\mathrm{cm}-)$ diameter quartz rod is admitted into the screen chamber through a 5/16-inch- $(0.79-\mathrm{cm}-)$ diameter aperture.

To monitor the gases present in the microscope envelope, a C.E.C. type 21-611 mass spectrometer was appended to the microscope near the screen chamber.

It should be mentioned that these experiments were performed under dynamic vacuum conditions, the background pressure being typically about $1 \times 10^{-8}$ torr, with water as the main contaminant.

It was found that quantities of neon-free helium imaging gas, large enough to supply the demands of the vacuum system could be obtained from the boiloff of a 50-liter $\left(5 \times 10^{4}-c c\right)$ liquid helium dewar. Other impurities, with the exception of hydrogen, were removed by passing the gas through a cold trap maintained at $4.2 \mathrm{~K}$. A titanium getter was attached to the gas handling system to remove hydrogen impurities, even though the mass spectrometer showed no detectable quantity of hydrogen. The blinking effect did not seem to be affected by the operation of the getter, indicating either that no hydrogen was present, or that, if it were, it had no effect on the blinking process.

Measurements of the blinking rate $(\mathrm{BR})$ of individual emitters were made by masking the screen and positioning a photomultiplier adjacent to the visible portion of the image.

The transition between the blinking mode and the stable imaging region (corresponding to the blinking cutoff voltage ( $\mathrm{BCOV})$ ) was detected by direct visual inspection of the screen with the aid of a low power microscope.

Although this phenomenon occurs on all observable planes, the measurements and analysis that follow are all concerned with the tungsten (111) plane because of ease of observation on this plane. 
The blinking effect was first noticed when the microscope was operated with commercial quality helium imaging gas with its attendant contaminants. It was subsequently found that the neon impurity was responsible for the blinking effect. The blinking phenomenon could be induced by the addition of only a few parts-per-million neon to pure helium imaging gas. Figure 2 shows the BR of one emitting atom on the (111) plane as a function of neon partial pressure at constant helium pressure. As can be seen from the figure, the data indicate a finite $B R$ even at zero neon partial pressure. The most obvious explanation of this residual blinking is that it is caused by diffusion from an adsorbed neon layer or film on the shank of the tip. In an attempt to remove the suspected layer, the field was removed for a few minutes. When the field was restored, the blinking continued. Next, with the voltage applied and pure helium gas present, the temperature of the tip was raised to about $30 \mathrm{~K}$ for a few minutes. The result was a cessation of blinking. Thus one concludes that the cause of residual blinking is the diffusion to the imaged portion of the tip of atoms from a mobile physisorbed phase on the shank of the tip that can be thermally desorbed at about $30 \mathrm{~K}$. Polarization forces do not seem to play a major part in the bonding of the adsorbed shank layer.

An adsorbed layer on the shank of the tip should deplete itself with time, and this was shown to be so. The BR was measured as a function of the time after the neon component of the imaging gas was removed and was found to decay with a time constant of the the order of 100 minutes.

The negligible effect of the helium imaging gas on the BR can be seen in figure 3 where the $\mathrm{BR}$ is plotted as a function of helium partial pressure for two different values of the neon partial pressure.

Figure 4 shows the variation of the $\mathrm{BR}$ with applied voltage at $5 \mathrm{~K}$ and $20 \mathrm{~K}$. The voltage limits for these measurements are (1) the BCOV at high voltage and (2) the onset of a bright, blurry emission mode at low voltage. The phenomenon occurring at the lower limit has been postulated (ref. 2) to be due to an enhancement of the supply of imaging gas atoms to the sharper (111) regions from the flatter regions around the (110) planes where the field is not high enough to produce significant ionization. As this limit (to be referred to as the BBV) is approached from higher voltages, the entire (111) plane suddenly becomes quite bright and the atom spots become large and diffuse. Because of this gross change in the appearance of the (111) plane, the BR measurements were terminated at the BBV, even though there appeared to be some blinking below this voltage.

The BCOV was found to be independent of both helium and neon partial pressures over the ranges $0.2 \times 10^{-3}<\mathrm{P}_{\mathrm{He}}<1.2 \times 10^{-3}$ torr and $5 \times 10^{-9}<\mathrm{P}_{\mathrm{Ne}}<5 \times 10^{-8}$ torr. The $\mathrm{BCOV}$ and the $\mathrm{BBV}$ are plotted as a function tip temperature in figure 5 . 


\section{DISCUSSION}

Once neon has been identified as the cause of blinking, the question arises whether the effect is due to the energetic impact of the gas-phase atoms with the surface, or whether the mere presence of neon on the surface is responsible for the blinking.

Because of the fact that blinking continues even after the gas-phase neon is removeo (fig. 2), one can conclude that the process is not due to energetic gas atom impact.

If, then, the presence of neon on the surface causes the blinking, there appear to be two alternatives. (1) The presence of the neon enhances the emission, producing an increase in brightness of an emitting atom. (2) The presence of neon causes a lessening of emission, observed as a temporary darkening of the image spot.

The latter possibility can be eliminated in light of the fact that atoms that are invisible when imaged with pure helium are periodically visible in the blinking mode. It is evident, therefore, that the action of the neon on these atoms is such as to increase the emission above them.

The location of the neon atoms while they are active in producing enhanced emission can be inferred from several qualitative observations. Two configurations readily come to mind.

First, neon atoms, adsorbing in the recesses or valleys between protruding substrate atoms, could conceivably cause electronic redistribution resulting in field enhancement above the substrate atoms. This type of mechanism has been postulated in the case of hydrogen on tungsten (ref. 3). It would not seem to be as likely a mechanism in the case of an inert gas, however.

Alternatively, an adsorbed atom on top of a protruding substrate atom (peak adsorption) would change the geometry in the vicinity of the emitter. The geometry change could result in field enhancement above the emitter complex which would produce enhanced emission.

The latter configuration is favored by the observation that the blinking process appears to involve only one atom at a time. One would expect that, for a valley adsorption complex, more than one substrate atom would be affected. A neon atom adsorbed between two tungsten atoms should, if it affected the electron distribution, cause field enhancement above both of its neighbors. Coordinated blinking of this sort has not been observed.

Peak adsorption, on the other hand, would affect only one emitter. Furthermore, if we can trust a calculation based on macroscopic electrostatics, it can be shown that the peak site is favored over the valley site energetically. The polarization induced binding energy, $1 / 2 \alpha \mathrm{F}^{2}$, of a neon atom adsorbed on a metal surface can be shown, for the field strengths of interest here, to be about an order of magnitude greater than the normal Van der Waals' adsorption energy which would be maximized at the valley sites. A field variation from a valley site to a peak site of only 5 percent would then be neces- 
sary to make the peak site energetically favorable.

One difficulty with the peak adsorption model is the need to explain the long ionization lifetimes in these high field regions. One recourse is to postulate a field-induced chemical bond that tightly bonds the neon atom to its peak site, thus preventing its movement above the critical distance $\mathrm{X}_{\mathrm{C}}$. Field-induced complexes of this sort have been reported in the literature. Mulson and Muller (ref. 4) have observed nitrogen atoms peak adsorbed on protruding tungsten substrate atoms. They report that, in some cases, the binding between the nitrogen and the tungsten atoms was so great as to cause the tungsten atom to be removed with the nitrogen atom when the nitrogen was field desorbed.

Furthermore, the desorption of neon-tungsten and helium-tungsten complexes has been observed by several investigators using the atom-probe time-of-flight mass spectrometer (refs. 5 and 6). More recently Tsong and Muller (ref. 7) have presented convincing evidence that, during the normal operation of the field-ion microscope, ionization takes place not over a bare tungsten atom, but over an inert gas atom peak adsorbed on the tungsten atom. Thus we must conclude that, in our model for the blinking effect, the field above a tungsten emitter is enhanced by the peak adsorption of a helium atom even before the arrival of a neon atom. When a neon atom does impinge upon the helium-tungsten complex, it would, because of its higher polarizability, be energetically favored to replace the helium atom. Because of its greater size and polarizability, the peak adsorbed neon atom would be expected to enhance the field to a greater extent than the peak adsorbed helium atom did. This greater enhancement would cause an increase in emission from the site, which would be seen on the screen as a blink.

Summarizing the previous paragraphs, then, the most feasible mechanism seems to involve the temporary replacement of a peak adsorbed helium atom with a similarly adsorbed neon atom. Because the neon-tungsten complex enhances the field to a greater extent than does the helium-tungsten complex, there is an observable increase in emission as long as the neon remains in this configuration.

A more detailed model of the blinking effect would have to explain, among other things, the strong field dependence of the blinking rate (fig. 4). The sharp fall off of the $\mathrm{BR}$ with rising field has several possible explanations, among which the following seem to be the most plausible:

(1) A field-dependent sticking probability model where the probability of a neon atom complexing with a tungsten emitter drops off rapidly with rising field strength

(2) A fast removal model, where, as the field is raised, an increasing percentage of the complexed adatoms are removed so quickly after adsorption that the resulting blinks are not observable because of experimental time-constant considerations. Two such mechanisms come to mind: (a) a gas-bombardment removal mechanism where the energy and impingement rate of the polarization-attracted gas atoms increase rapidly with field and (b) a field-desorption removal model. 
(3) A mechanism which involves the reduction, with rising field, of the probability of neutral neon atom penetration of the ionization zone above an emitter

Of these possibilities, only the last, the penetration probability mechanism, has the correct temperature dependence. Both the sticking probability mechanism and the quick removal mechanism are thermally assisted in such a way that at higher temperatures they predict lower blinking rates, in conflict with experimental results.

The penetration probability mechanism is not without difficulties, however. According to the literature (ref. 8), the transition between ionization-limited operation of the tungsten-neon F.I.M. and supply limited operation occurs at a field of about 2.75 volts per $\AA$. If we interpret the blinking rate data as being due to a penetration probability mechanism, then the sharp field dependence of the BR (fig. 4) suggests that the process is still ionization limited at fields as high as 4 volts per $\AA$.

The situation is even worse when one considers the fact that the field above an emitter is enhanced by the presence of a peak adsorbed helium atom. This enhancement should make it easier to ionize the incoming neon atoms, thus making it more difficult to understand the ionization limited behavior in this high field region. The reason for this behavior is not clearly understood, but the following explanation gives results that are consistent with the experimental data.

It will be assumed in the following that, at fields sufficiently above $F_{c}$ (i.e., the transition field between ionization limited operation and supply limited operation), a typical emitter configuration consists of a helium-tungsten peak adsorption complex. A field enhancement factor $\sigma$ will be used to describe the field over a complex, such that

$$
\mathbf{F}^{\prime}=\sigma \mathbf{F}
$$

where $F^{\prime}$ is the enhanced field above an emitter complex and $F$ is the field to be expected over a noncomplexed tungsten emitter.

It will also be assumed that

(1) Even if a neon atom is singly ionized during its passage through the ionization zone above an emitter, it still has the ability to replace the complexed helium adatom and produce a blink if the conditions of the collision are right.

(2) If the neon atom is doubly ionized on its passage through the zone, it has a negligible probability of replacing the complexed helium.

Let us now derive an expression for the blinking rate by postulating that

$$
\mathrm{BR}=\beta \mathrm{N}
$$

where $\mathrm{N}$ is the number of neon atoms that pass through the ionization zone above an emitter per second without being doubly ionized and $\beta$ is the probability of peak adsorption per such pass. This model assumes that, as neon atoms approach an emitter complex, they pass through the ionization zone above the emitter. During their pass through 
the zone, some of the neon atoms are doubly ionized and do not complex with the tungsten emitter. Of the remaining atoms, a fraction $\beta$ complex with the tungsten atom causing enhanced emission which is observed as a blink.

The probability of ionization, per pass through the ionization zone, per atom, can be given in terms of the ionization lifetime $\tau$ and the transit time through the zone $\Delta t$ by the expression

$$
P_{I}=1-\exp -\frac{\Delta t}{\tau}
$$

The probability of passing through the zone without ionization is therefore

$$
P_{0}=1-P_{I}=\exp -\frac{\Delta t}{\tau}
$$

If $\nu$ is the number of passes attempted per atom, per second, and $\mathrm{C}$ is the number of atoms attempting to pass through the zone, then the number of nonionizing passes through the zone per second is

$$
\mathrm{N}=\mathrm{C} \nu \exp -\frac{\Delta \mathrm{t}}{\tau}
$$

If $\mathrm{Z}$ is the arrival rate of neon atoms to the emitter of interest, and $\mathrm{k}_{\mathrm{i}}$ and $\mathrm{k}_{\mathrm{d}}$ are the rate constants for removal from the plane of interest by ionization and by means other than ionization, respectively, then

$$
\mathrm{C}=\frac{\mathrm{Z}}{\mathrm{k}_{\mathrm{i}}+\mathrm{k}_{\mathrm{d}}}
$$

The ionization rate constant can be written

$$
\mathrm{k}_{\mathrm{i}}=\nu \mathrm{P}_{\mathrm{I}}=\nu\left(1-\exp -\frac{\Delta t}{\tau}\right)
$$

Combining equations (5), (6), and (7) yields an expression for $N$

$$
\mathrm{N}=\frac{\mathrm{Z}}{\left(1+\frac{\mathrm{k}_{\mathrm{d}}}{\nu}\right) \exp \frac{\Delta \mathrm{t}}{\tau}-1}
$$

From equations (2) and (8) the blinking rate is 


$$
\mathrm{BR}=\frac{\beta \mathrm{Z}}{\left(1+\frac{\mathrm{k} d}{\nu}\right) \exp \frac{\Delta \mathrm{t}}{\tau}-1}
$$

The supply function $\mathrm{Z}$ is a complicated function of the tip temperature. From brightness measurements taken at the BIV (ref. 8), it has been shown that the overall supply function increases rapidly as the tip temperature is lowered. This probably indicates an increase, with decreasing temperature, in the sticking coefficient for incoming gas atoms on the shank of the tip or a decrease in the thermal desorption rate for gas atoms diffusing from the shank to the imaging region, or both.

Further complications arise when one considers the supply function to a given plane. Muller, Nakamura, Nishikawa, and McLane (ref. 2) have shown that, for helium imaging gas, the supply function to the sharper regions around the (111) plane depends upon the ability of the flatter regions around the (110) planes to ionize their supply of imaging gas efficiently. Although similar data for neon have not been published, it is likely that it would act similarly.

The lack of information concerning these factors makes a theoretical calculation of the variation of the supply function, and thus the blinking rate, with temperature impossible at present.

On the other hand, while the field variation of the supply function to the (111) plane is equally difficult to estimate, the extremely strong field dependence of the denominator of equation (9) permits us to ignore the field variation of $\mathrm{Z}$ without introducing significant error into the results.

The supply function $\mathrm{Z}$ or more correctly, the product $\beta \mathrm{Z}$ will therefore be treated as an adjustable parameter, independent of field, in an attempt to fit equation (9) to the experimental data.

At the transition field $F_{c}$ where the mode of operation of the F.I.M. changes from ionization limited to supply limited, the transit time through the ionization zone $\Delta t$ is equal to the ionization time $\tau$. Using Gomer's approximation for $\tau$ (ref. 9) gives

$$
\tau=10^{-16} \exp \left[0.68 \frac{\mathrm{I}-\varphi}{\mathrm{F}}\left(\mathrm{I}-7.6 \mathrm{~F}^{1 / 2}\right)^{1 / 2}\right] \quad \mathrm{sec}
$$

where $I$ is the imaging gas ionization potential in $\mathrm{eV}, \varphi$ is the work function of the tip in $\mathrm{eV}$, and $\mathrm{F}$ is the field in volts per $\AA$. Noting that for neon at $78 \mathrm{~K}, \mathrm{~F}_{\mathrm{c}} \cong 2.75$ volts per $\AA$, we can get a consistent value of $\Delta t$

$$
\tau_{\mathrm{c}}=\Delta \mathrm{t}=3.23 \times 10^{-11} \mathrm{sec}(\text { at } 78 \mathrm{~K})
$$

The values of the parameters used are $\mathrm{I}=21.6 \mathrm{eV}$ and $\varphi=4.5 \mathrm{eV}$. 
Assuming that $\Delta t$ is independent of field to a first approximation, we can correct for temperature by noting, with Brandon ( $r$ ef. 10), that

$$
\Delta t \propto \mathbf{T}^{-1 / 2}
$$

Values obtained for $\Delta \mathrm{t}$ at 5 and $20 \mathrm{~K}$ are given in table $\mathrm{I}$.

If we assume with Muller (ref. 11) that the means of removal from the tip, other than by ionization, consists of the diffusion of adatoms to the shank from the ernitting region, we obtain for $\mathrm{k}_{\mathrm{d}}$

$$
\mathrm{k}_{\mathrm{d}}=\frac{4 \sqrt{2}(\mathrm{kT})^{3 / 2}}{n M^{1 / 2} \mathrm{r} \alpha \mathrm{F}^{2}}
$$

where $\mathrm{M}$ and $\alpha$ are the gas atom mass and polarizability, respectively, $r$ is the tip radius, and $\mathrm{n}$ is a geometry factor.

Similarly, from Muller (ref. 11)

$$
\nu=\frac{\mathrm{n} \alpha \mathrm{F}^{2}}{2 \mathrm{r}(2 \mathrm{kTM})^{1 / 2}}
$$

Thus,

$$
\frac{\mathrm{k}_{\mathrm{d}}}{\nu}=\left(\frac{4 \mathrm{kT}}{\ln \alpha \mathrm{F}^{2}}\right)^{2}
$$

Making the transformation from field to voltage through the relation $a=F / V$ results in

$$
\frac{\mathrm{k}_{\mathrm{d}}}{\nu}=\left(\frac{4 \mathrm{kT}}{\mathrm{n} \alpha \mathrm{a}^{2} \mathrm{v}^{2}}\right)^{2} \equiv \frac{\gamma}{\mathrm{v}^{4}}
$$

If $F=4.5$ volts per $\AA$ when $V=5.6$ kilovolts (BIV in fig. 4), then $a=0.805 \AA^{-1}$. Using $\mathrm{n}=4 / 3$ and $\alpha=3.92 \times 10^{-25} \mathrm{~cm}^{3}$, values of $\gamma$ at 5 and $20 \mathrm{~K}$ have been calculated and are listed in table $I$.

If we combine equations (9), (10), and (11) and introduce the factor $\sigma$ through equation (1) (i.e., use $F^{\prime}$ in place of $F$ to account for the field enhancing effects of the helium-tungsten complex), we obtain an expression for the BR as a function of the applied voltage. In this expression the second ionization potential for neon $(\sim 41 \mathrm{eV})$ must be used in accordance with the assumption that double ionization of approaching neon atoms precludes their complexing with the protruding tungsten substrate atoms. If the parameters $\sigma$ and $\beta \mathrm{Z}$ are adjusted so as to give the best fit to the experimental 
data of figure 4, we obtain the solid curves in that figure. As can be seen, the agreement with experiment is good, and $\sigma$ and $\beta \mathrm{Z}$ take on reasonable values which are given in table I for tip temperatures of 5 and $20 \mathrm{~K}$.

The lifetime of a complexed neon adatom, as measured by the area under the brightness peak, seems to be related to the number and energy of the atoms striking the surface from the gas phase. The following observations have been made:

(1) The measured brightness peak area increases with decreasing helium imaging gas pressure.

(2) The area increases as heavy impurities, such as nitrogen, are removed from the imaging gas.

(3) The area decreases as the voltage is raised toward BCOV. The number and energy of gas-phase atoms striking the surface increase with the applied voltage.

This last consideration may explain the sharp cutoff in the blinking rate at the BCOV where equation (9) predicts a gradual fall-off. It is suggested that, near BCOV, the lifetimes of the peak adsorbed neon atoms become so short that the experimental time constants preclude detection of the peaks.

\section{SUMMARY OF RESULTS}

The results of these investigations into the cause of the blinking effect can be summarized as follows:

1. A blink or brightness pulse has been found to be due to the momentary adsorption of a neon atom on the apex of a tungsten substrate atom. The resulting protrusion causes field enhancement and thus an increase of emission from the site.

2. The blinking rate on the (111) plane has been shown to be proportional to the probability that a neon atom will penetrate the ionization zone above an emitter without being doubly ionized.

3. Below $30 \mathrm{~K}$, a mobile neon layer is formed on the shank of the tip. Diffusion from this layer to the imaged portion of the tip seems to be the major source of supply of neon to the cap of the tip.

4. The removal of peak adsorbed neon is at least partially due to the impact of energetic gas-phase atoms.

Lewis Research Center,

National Aeronautics and Space Administration,

Cleveland, Ohio, November 30, 1970,

129-03. 


\section{REFERENCES}

1. Weizer, Victor G.; and Forestieri, Americo F.: On the Resolution and Image Intensity of the Field-Ion Microscope. NASA TN D-5125, 1969.

2. Miiller, E. W.; Nakamura, S.; Nishikawa, O.; and McLane, S. B.: Gas-Surface Interactions and Field-Ion Microscopy of Nonrefractory Metals. J. Appl. Phys., vol. 36, no. 8, Aug. 1965, pp. 2496-2503.

3. Muller, Erwin W.: Hydrogen Promotion of Field Ionization and Rearrangement of Surface Charge. Surface Sci., vol. 7, no. 3, 1967, pp. 462-473.

4. Mulson, J. F.; and Müller, E. W.: Corrosion of Tungsten and Iridium by Field Desorption of Nitrogen and Carbon Monoxide. J. Chem. Phys., vol. 38, no. 11, June 1, 1963, pp. 2615-2619.

5. McKinney, J. T.; and Brenner, S. S.: Atom Probe Study of Field Evaporation In the Presence of an Imaging Gas. Presented at the 16th Field Emission Symposium, Pittsburgh, Pa., 1969.

6. Müller, E. W. ; Mclane, S. B. ; and Panitz, J. A.: Field Adsorption and Desorption of Helium and Neon. Presented at the 16th Field Emission Symposium, Pittsburgh, Pa., 1969.

7. Tsong, Tien T.; and Müller, Erwin W.: Field Adsorption of Inert-Gas Atoms on Field Icn Emitter Surfaces. Phys. Rev. Letters, vol. 25, no. 14, Oct. 5, 1970, pp. 911-913.

8. Tsong, Tien Tzou; and Müller, Erwin W.: Current-Voltage Characteristics by Image Photometry in a Filled-Ion Microscope. J. Appl. Phys., vol. 37, no. 8, July 1966, pp. 3065-3070.

9. Gomer, Robert: Field Emission and Field Ionization. Harvard Univ. Press, 1961, p. 78 .

10. Brandon, D. G.: The Resolution of Atomic Structure: Recent Advances in the Theory and Development of the Field Ion Microscope. Brit. J. Appl. Phys. , vol. 14, Aug. 1963, pp. 474-484.

11. Müller, Erwin W.; and Tsong, T. T.: Field Ion Microscopy. American Elsevier Publ. Co., 1969. 
TABLE I. - VALUES OF PARAMETERS USED IN EQUATION (9)

\begin{tabular}{|c|c|c|c|c|}
\hline $\begin{array}{c}\text { Tip } \\
\text { temperature, } \\
\text { K }\end{array}$ & $\begin{array}{c}\text { Transit time } \\
\text { through } \\
\text { ionization } \\
\text { zone, } \\
\Delta t, \\
\text { sec }\end{array}$ & $\begin{array}{c}\gamma, \\
\text { volts }\end{array}$ & $\begin{array}{c}\text { Field enhance- } \\
\text { ment factor, } \\
\sigma\end{array}$ & $\begin{array}{c}\beta Z, \\
\text { sec }^{-1}\end{array}$ \\
\hline 5 & $1.275 \times 10^{-10}$ & $3.07 \times 10^{-3}$ & 1.227 & $1.626 \times 10^{-5}$ \\
20 & $6.37 \times 10^{-11}$ & $4.91 \times 10^{-2}$ & 1.325 & $2.641 \times 10^{-4}$ \\
& & &
\end{tabular}

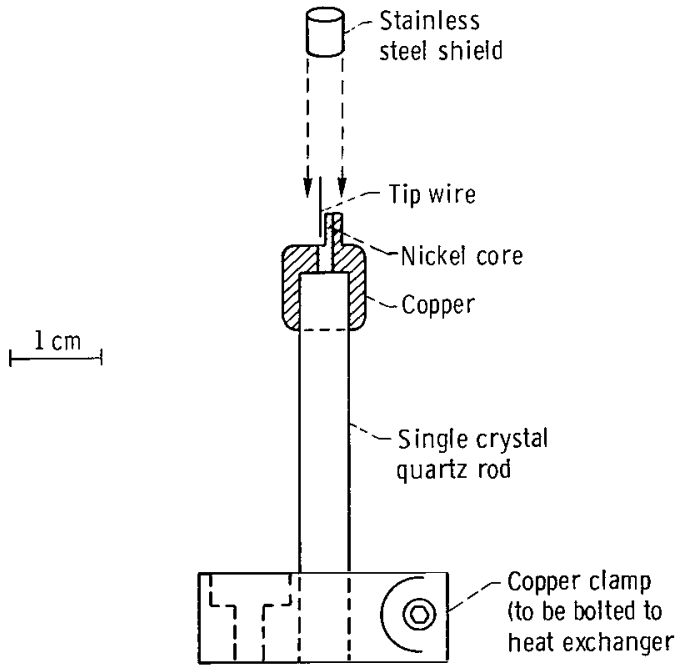

Figure 1. - Diagram of modified sample holder.

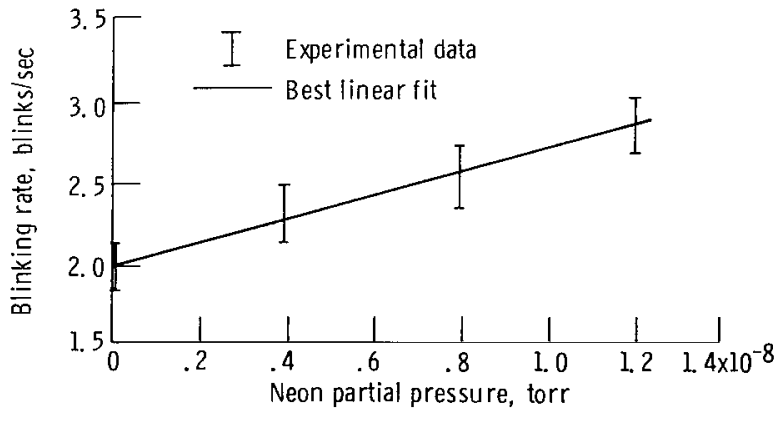

Figure 2. - Plot of blinking rate of single emitter on (111) plane as a function of neon partial pressure. Helium partial pressure, $6 \times 10^{-4}$ torr; tip temperature, $5 \mathrm{~K}$. (Pressures are gage readings - not corrected for gas species.) 


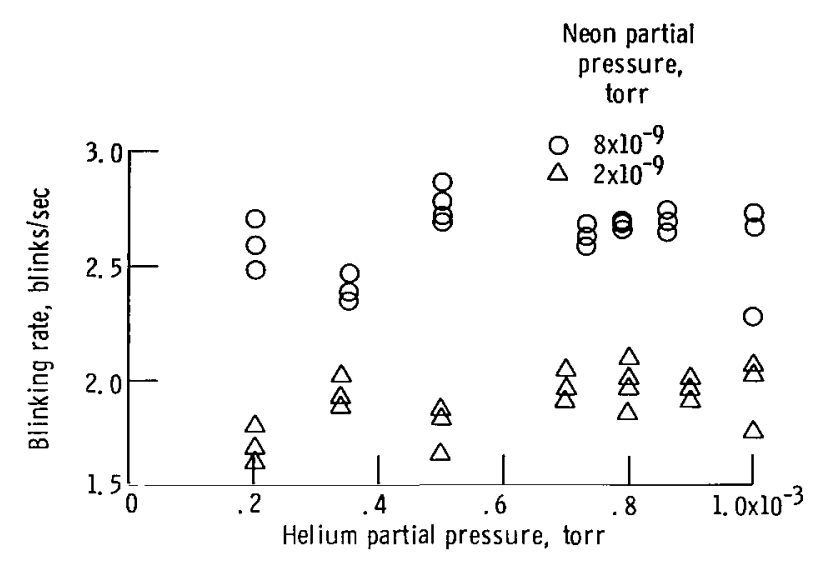

Figure 3. - Plot of blinking rate as a function of helium pressure for two neon partial pressures. Tip temperature, $5 \mathrm{~K}$.

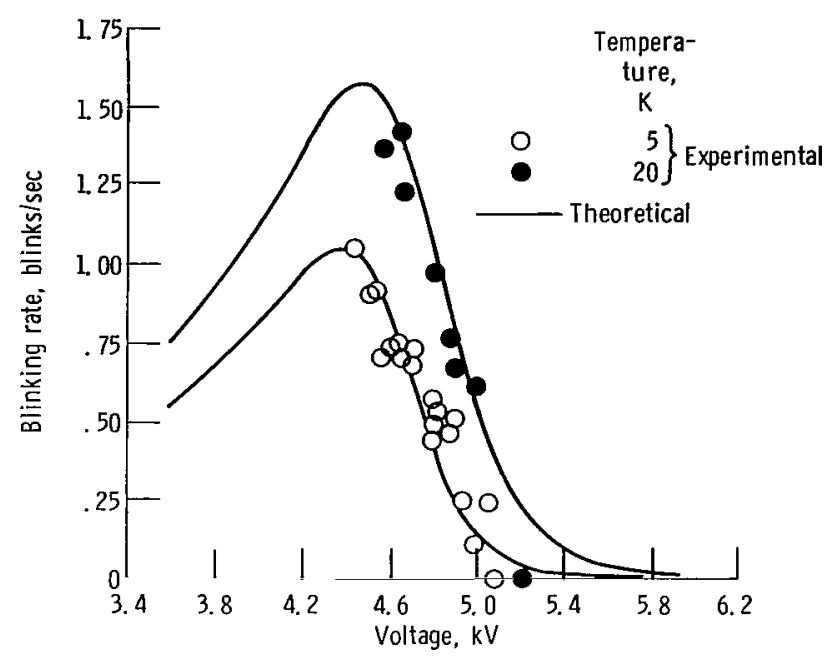

Figure 4. - Plot of blinking rate as a function of applied voltage for tip temperatures of 5 and $20 \mathrm{~K}$. Helium partial pressure, $7.8 \times 10^{-4}$ torr; neon partial pressure, $2 \times 10^{-8}$ torr.

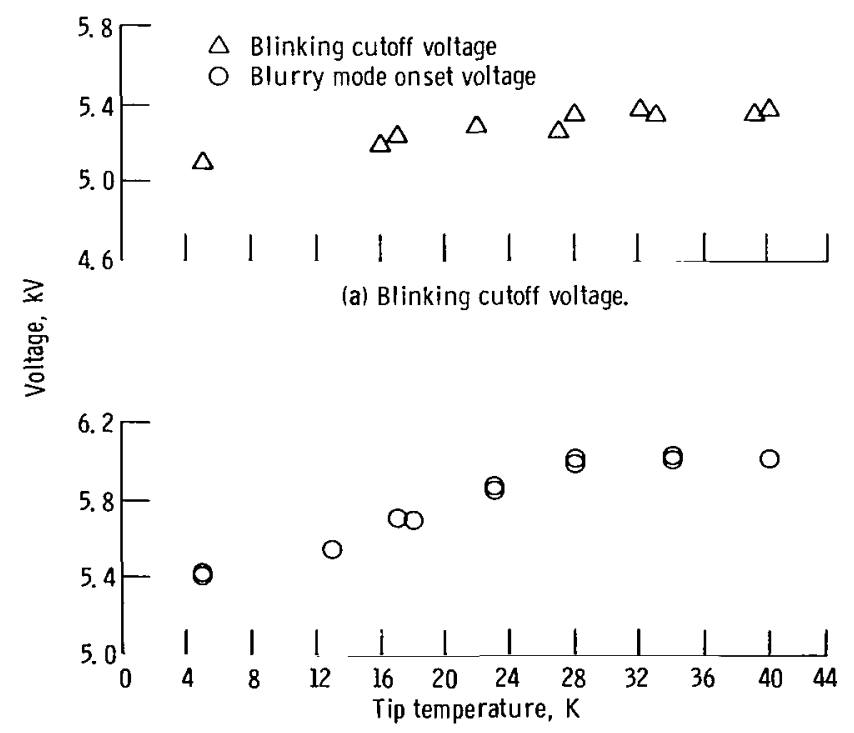

(b) Blurry mode onset voltage.

Figure 5. - Plot of blinking cutoff voltage ( $\mathrm{BCOV}$ ) and blurry mode on set voltage (BBV) as a function of tip temperature. (These two sets of data do not refer to same tip.) 


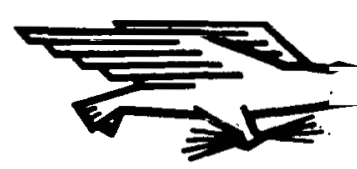

POSTAGE AND FEES PAI! NATIONAL AERONAUTICS SPACE ADMINISTRATION

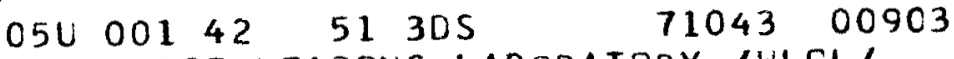 \\ AIR FORCE WEAPONS LABORATORY /WLCL/ \\ KIRTLAND AFB, NEW MEXICO 87117 \\ ATT E. LOU BOWMAN, CHIEF, TECH. LIBRARY
}

POSTMASTER: If Undeliverable (Section 1

"The deronautical and space activities of the United States shall be conducted so as to contribute ... to the expansion of buman knowledge of phenomena in the atmosphere and space. The Administration shall provide for the widest practicable and appropriate dissemination of information concerning its activities and the results thereof."

- National Aeronautics and Space ACt of 1958

\title{
NASA SCIENTIFIC AND TECHNICAL PUBLICATIONS
}

TECHNICAL REPORTS: Scientific and technical information considered important, complete, and a lasting contribution to existing knowledge.

TECHNICAL NOTES: Information less broad in scope but nevertheless of importance as a contribution to existing knowledge.

TECHNICAL MEMORANDUMS:

Information receiving limited distribution because of preliminary data, security classifica. tion, or other reasons.

CONTRACTOR REPORTS: Scientific and technical information generated under a NASA contract or grant and considered an important contribution to existing knowledge.
TECHNICAL TRANSLATIONS: Information published in a foreign language considered to merit NASA distribution in English.

SPECIAL PUBLICATIONS: Information derived from or of value to NASA activities. Publications include conference proceedings, monographs, data compilations, handbooks, sourcebooks, and special bibliographies.

\section{TECHNOLOGY UTILIZATION}

PUBLICATIONS: Information on technology used by NASA that may be of particular interest in commercial and other non-aerospace applications. Publications include Tech Briefs, Technology Utilization Reports and Technology Surveys.

Details on the availability of these publications may be obtained from:

SCIENTIFIC AND TECHNICAL INFORMATION OFFICE

NATIONAL AERONAUTICS AND SPACE ADMINISTRATION Washington, D.C. 20546 